\title{
LEAD-TIME, INVENTORY, AND SAFETY STOCK CALCULATION IN JOB-SHOP MANUFACTURING
}

\author{
Miguel Afonso Sellitto \\ Universidade do Vale do Rio dos Sinos, Production and Systems Engineering Graduate Program, Av. Unisinos 950, \\ São Leopoldo RS 93022-000 Brazil \\ correspondence: sellitto@unisinos.br
}

\begin{abstract}
The purpose of this article is to present a method for calculating the lead-time, the inventory, and the safety stock or buffer in job shop manufacturing, which are essentially stochastic variables. The research method is quantitative modelling. The theoretical foundation of the method relies on the techniques belonging to the WLC (workload control) body of knowledge on the manufacturing management. The study includes an application of the method in a manufacturing plant of the furniture industry, whose operation strategy requires high dependability. The company operates in a supply chain and must have high reliability in deliveries. Adequate safety stocks, lead times, and inventory levels provide the protection against the lack of reliability in the deliveries. The inventory should remain within a certain range, being as small as possible to maintain low lead times, but not too small that it could provoke a starvation, configuring an optimization problem. The study offers guidelines for a complete application in industries. Further research shall include the influence of the variability of the lot size in the stochastic variables.
\end{abstract}

KEYWORDS: lead-time; inventory; safety stock; dependability; workload control; queuing theory.

\section{INTRODUCTION}

Workload Control (WLC) is a production planning and control technique suitable for high-variety job shop manufacturing [1] and focused mainly on Make-toOrder (MTO) production [2]. The WLC integrates two control mechanisms, the input and the output control. The input control regulates the income of workload into the manufacturing system by priority rules. The output control regulates the outcome of the orders by adjustments in the production capacity. The extant literature considers the load-based order release as the main input control technique and adding or removing equipment and workforce as the main output control technique [1. The dependent variable is the lead-time, the time between the arrival and the completion of an order, controlled by the work-in-process inventory, the orders waiting for service. Small inventories decrease the time an order waits for service, decreasing the lead-time.

This study focuses on the Southern Brazilian furniture industry. The main strategic objective of the industry is a high dependability, which includes high speed (short due dates), high reliability (accuracy in quantity and quality), and on-time deliveries (no tardiness). In the furniture industry, the manufacturing operates mostly in job shop plants based on the MTO production. The industry produces furniture items and mattresses and covers activities that range from the extraction and reception of raw materials from suppliers to the delivery, installation, and technical assistance of goods. A typical manufacturing company designs and produces a high variety of items with wood, steel, plastic, and specific materials. The industry must comply with various and rigorous technical specifications, whose application may require, at the same time, the use of advanced technologies and artisanal skills. The innovation depends mainly on the development of new materials and new design techniques, in a close cooperation with suppliers 3 . The main elements of a furniture design are plates, fasteners, paints and resins, upholstery, and packaging. Some products, like rectilinear items, support a high automation level, while others, like solid wood items, require artisan skills 4 .

Although the WLC originally focused on highly balanced production lines, further results indicate that the WLC could also be effective in unbalanced situations, like those found in the furniture industry. Not only bottlenecks but also non-bottlenecks work centres receive close attention 2 . Otherwise, the WLC performance might strongly deteriorate if the protective capacity of critical resources is not sufficiently high [5]. Despite most reported implementations are in large manufacturing plants, the WLC is particularly suitable for Small and Medium-sized Enterprises 6, such as those found in the furniture industry. In the furniture industry, a large part of the production comes from small and medium size plants, organized in the job-shop MTO production, which makes the WLC a useful tool in the manufacturing control function [7].

The purpose of this article is to present a method for calculating the lead-time, the inventory, and the safety stock in a job shop MTO manufacturing. The research method is quantitative modelling. The theoretical foundation of the method relies on proper contributions and on some issues extracted from the WLC body of knowledge. The research object is a manufacturing plant of the furniture industry of Southern Brazil. The company aims at a high dependability and must rely on adjusted levels of safety stocks, lead-times, and inventory levels to ensure on-time deliveries. The decision on inventory levels in the manufacturing stems from risk hedge policies due to unreliable suppliers, seasonal 
production, short-term deliveries, or low-consumption items. The calculation of the inventory level creates an optimization problem. If a given level of inventories ensures a satisfaction of some specific customers, it also entails financial costs to maintain the cash flow [1618. The remainder of the article contains the review, methodology, results, discussion, and conclusions.

\section{Theoretical BaCKGround}

The WLC or Load-Oriented Control aims at controlling the manufacturing workload controlling the inventory level [8]. Over time, the WLC balances the workload releases to and from the manufacturing system. As inventory does not grow above a given level, the lead-time remains low. As the inventory does not fall below a given level, efficiency remains high 9. As the MTO manufacturing works with low quantity and high variety production, the control requires stochastic approaches [10].

The model employs five stochastic variables: lead-time $(L T)$, work-in-process $(W I P)$, arrival rate $(R I)$, throughput $(P)$, and safety stock $(S S)$. The $L T$ (measured in days or hours) varies according to the time waiting for service and the processing time. Order $L T$ considers only the time to completion. Part $L T$ considers also the size of the orders. WIP (measured in parts, tons, or other units) is the queue in the manufacturing system and represents the instantaneous quantity of materials waiting for service in the shop-floor. $R I$ is the rate of arrival of orders, measured in pieces or tons per day or per hour. $P$ is the rate of orders or parts delivery, measured by the same unit as $R I$. Imbalances between $R I$ and $P$ modify the queue length and, consequently, the WIP. Finally, $S S$ is the minimum level of WIP that, under a given confidence level, prevents the starvation produced by instantaneous differences between $R I$ and $P[8,12,13$. The calculation model is valid for an isolated work centre as well as for a complete production line [8], and it looks as follows:

$$
\begin{aligned}
L T_{i} & =T P E U_{i}-T P E_{i}, \\
L T_{\mathrm{m}} & =\sum_{i=1}^{n} \frac{L T_{i}}{n}, \\
L T_{\sigma \mathrm{m}} & =\sqrt{\frac{\sum_{i=1}^{n}\left(L T_{\mathrm{m}}-L T_{i}\right)^{2}}{n-1},} \\
L T_{\mathrm{mw}} & =\frac{\sum_{i=1}^{n} L T_{i} Q_{i}}{\sum_{i=1}^{n} Q_{i}}, \\
L T_{\sigma \mathrm{w}} & =\sqrt{\frac{\sum_{i=1}^{n}\left(L T_{\mathrm{m}}-L T_{i}\right)^{2} Q_{i}}{\sum_{i=1}^{n} Q_{i}}}, \\
R I_{\mathrm{m}} & =\frac{\sum_{i=1}^{n} Q_{i}}{\max T P I_{i}-\min T P I_{i}}, \\
W I P_{\mathrm{m}} & =R I_{\mathrm{m}} L T_{\mathrm{mw}}, \\
R I_{\mathrm{m}} & =\frac{\sum_{i=1}^{n} Q_{i}}{\max T P E U_{i}-\min T P E U_{i}}, \\
S S_{\mathrm{m}} & =P_{\mathrm{m}} \Delta T_{\max },
\end{aligned}
$$

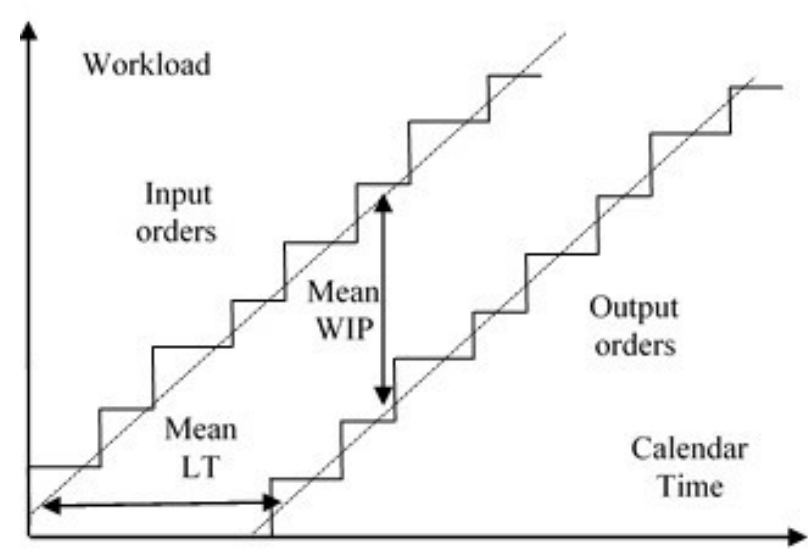

Figure 1. The Throughput Diagram.

in which: $L T_{i}$ - lead-time $(L T)$ of order $i$ in the current work centre; $T P E_{i}-$ completion time of order $i$ in the previous work centre (time of liberation); TPEU $U_{i}$ completion time of order $i$ in the current work centre. $n$ - number of orders. $L T_{i}$ - lead-time of order $i$; $L T_{\mathrm{m}}$ - mean order $L T ; L T_{\sigma \mathrm{m}}$ - standard deviation of the order $L T ; L T_{\mathrm{mw}}$ - mean part $L T ; Q_{i}$ - amount of value added by order $i ; \sum_{i=1}^{n} Q_{i}$ - total amount dispatched by all orders; $L T_{\sigma \mathrm{w}}-$ standard deviation of the part $L T ; R I_{\mathrm{m}}$ - mean arrival rate of orders or parts; $W I P_{\mathrm{m}}$ - mean WIP; $P_{\mathrm{m}}$ - mean throughput; $\Delta T_{\max }-$ maximum time elapsed between the arrival of two successive orders.

The Throughput Diagram (TD) derives from the queue theory and represents the materials' flow through the manufacturing system. The TD is a graphical method to verify the analytical calculation of the LT and WIP [7, 8]. In the shop-floor, the TD also helps with the control of the queue, keeping $L T$ short and stable over time [2, 12]. Figure 1] illustrates the TD.

\section{Methodology AND ReSUlts}

The research procedures are:

(1.) data collection in a company of the furniture industry that manufactures desk-chair sets for offices (one set contains one or two chairs and one desk);

(2.) application of the calculation model;

(3.) analysis of the results regarding strategic priorities.

The company operates an MTO job shop manufacturing that processes orders with a size of multiples of ten. Eventual surplus routs to distribution centres for fast delivery. The company's manufacturing information system provided the data from the first hundred orders served in 2018. The company uses a Manufacturing Execution System (MES) to control the orders. The information retrieved from the MES are the size of the order, the release date, and the completion date. Tables 1 and 2 show the raw data and the results of the application respectively. 


\begin{tabular}{|c|c|c|c|c|c|c|c|}
\hline Order \# & $Q_{i}($ sets $)$ & $T P E_{i}$ & $T P E U_{i}$ & $L T_{i}$ & $Q_{i} L T_{i}$ & $\left(L T_{\mathrm{mw}}-L T_{i}\right)^{2}$ & $\left(L T_{\mathrm{mw}}-L T_{i}\right)^{2} Q_{i}$ \\
\hline 1 & 20 & $02 / 01$ & $04 / 01$ & 2 & 40 & 88.6 & 1772.6 \\
\hline 2 & 40 & $02 / 01$ & $17 / 01$ & 15 & 600 & 12.9 & 514.3 \\
\hline 3 & 50 & $02 / 01$ & 03/01 & 1 & 50 & 108.5 & 5423.0 \\
\hline 4 & 30 & $02 / 01$ & $15 / 01$ & 13 & 390 & 2.5 & 75.4 \\
\hline 5 & 70 & $03 / 01$ & $21 / 01$ & 18 & 1260 & 43.4 & 3035.9 \\
\hline 6 & 30 & $03 / 01$ & $22 / 01$ & 19 & 570 & 57.5 & 1726.2 \\
\hline 7 & 30 & $04 / 01$ & $24 / 01$ & 20 & 600 & 73.7 & 2211.4 \\
\hline 8 & 40 & $04 / 01$ & $14 / 01$ & 10 & 400 & 2.0 & 80.0 \\
\hline 9 & 30 & $07 / 01$ & 08/01 & 1 & 30 & 108.5 & 3253.8 \\
\hline 10 & 80 & $07 / 01$ & $18 / 01$ & 11 & 880 & 0.2 & 13.7 \\
\hline 11 & 30 & 08/01 & $17 / 01$ & 9 & 270 & 5.8 & 174.9 \\
\hline 12 & 30 & 09/01 & $15 / 01$ & 6 & 180 & 29.3 & 879.5 \\
\hline 13 & 30 & $10 / 01$ & $17 / 01$ & 7 & 210 & 19.5 & 584.6 \\
\hline 14 & 60 & $10 / 01$ & $17 / 01$ & 7 & 420 & 19.5 & 1169.2 \\
\hline 15 & 40 & $11 / 01$ & $21 / 01$ & 10 & 400 & 2.0 & 80.0 \\
\hline 16 & 30 & $11 / 01$ & $30 / 01$ & 19 & 570 & 57.5 & 1726.2 \\
\hline 17 & 60 & $14 / 01$ & $02 / 02$ & 19 & 1140 & 57.5 & 3452.4 \\
\hline 18 & 40 & $15 / 01$ & $01 / 02$ & 17 & 680 & 31.2 & 1247.9 \\
\hline 19 & 90 & $16 / 01$ & $27 / 01$ & 11 & 990 & 0.2 & 15.5 \\
\hline 20 & 30 & $16 / 01$ & $06 / 02$ & 21 & 630 & 91.9 & 2756.5 \\
\hline 21 & 80 & $21 / 01$ & $04 / 02$ & 14 & 1120 & 6.7 & 534.8 \\
\hline 22 & 20 & $22 / 01$ & $27 / 01$ & 5 & 100 & 41.1 & 822.9 \\
\hline 23 & 20 & $24 / 01$ & $28 / 01$ & 4 & 80 & 55.0 & 1099.5 \\
\hline 24 & 20 & $25 / 01$ & $28 / 01$ & 3 & 60 & 70.8 & 1416.1 \\
\hline 25 & 20 & $25 / 01$ & $22 / 02$ & 28 & 560 & 275.1 & 5501.6 \\
\hline 26 & 50 & $28 / 01$ & $13 / 02$ & 16 & 800 & 21.0 & 1051.4 \\
\hline 27 & 20 & $30 / 01$ & $31 / 01$ & 1 & 20 & 108.5 & 2169.2 \\
\hline 28 & 90 & $01 / 02$ & 08/02 & 7 & 630 & 19.5 & 1753.9 \\
\hline 29 & 40 & $04 / 02$ & $14 / 02$ & 10 & 400 & 2.0 & 80.0 \\
\hline 30 & 30 & $04 / 02$ & $06 / 02$ & 2 & 60 & 88.6 & 2658.9 \\
\hline 31 & 20 & $04 / 02$ & $07 / 02$ & 3 & 60 & 70.8 & 1416.1 \\
\hline 32 & 30 & $04 / 02$ & $20 / 02$ & 16 & 480 & 21.0 & 630.8 \\
\hline 33 & 20 & $04 / 02$ & $22 / 02$ & 18 & 360 & 43.4 & 867.4 \\
\hline 34 & 40 & $05 / 02$ & $13 / 02$ & 8 & 320 & 11.7 & 466.3 \\
\hline 35 & 40 & $05 / 02$ & $14 / 02$ & 9 & 360 & 5.8 & 233.2 \\
\hline 36 & 40 & $05 / 02$ & $16 / 02$ & 11 & 440 & 0.2 & 6.9 \\
\hline 37 & 30 & $05 / 02$ & $06 / 02$ & 1 & 30 & 108.5 & 3253.8 \\
\hline 38 & 50 & $05 / 02$ & $19 / 02$ & 14 & 700 & 6.7 & 334.3 \\
\hline 39 & 20 & $05 / 02$ & $22 / 02$ & 17 & 340 & 31.2 & 624.0 \\
\hline 40 & 70 & $05 / 02$ & $25 / 02$ & 20 & 1400 & 73.7 & 5159.8 \\
\hline 41 & 30 & $06 / 02$ & $18 / 02$ & 12 & 360 & 0.3 & 10.3 \\
\hline 42 & 50 & $06 / 02$ & $20 / 02$ & 14 & 700 & 6.7 & 334.3 \\
\hline 43 & 30 & $06 / 02$ & $22 / 02$ & 16 & 480 & 21.0 & 630.8 \\
\hline 44 & 20 & $07 / 02$ & $14 / 02$ & 7 & 140 & 19.5 & 389.7 \\
\hline 45 & 20 & $07 / 02$ & $14 / 02$ & 7 & 140 & 19.5 & 389.7 \\
\hline 46 & 60 & $07 / 02$ & $19 / 02$ & 12 & 720 & 0.3 & 20.6 \\
\hline 47 & 20 & $07 / 02$ & $19 / 02$ & 12 & 240 & 0.3 & 6.9 \\
\hline 48 & 50 & $07 / 02$ & $25 / 02$ & 18 & 900 & 43.4 & 2168.5 \\
\hline 49 & 60 & $07 / 02$ & $25 / 02$ & 18 & 1080 & 43.4 & 2602.2 \\
\hline 50 & 80 & $07 / 02$ & $21 / 02$ & 14 & 1120 & 6.7 & 534.8 \\
\hline
\end{tabular}

TABle 1. Raw data and the model application. Part $1 / 2$. 


\begin{tabular}{|c|c|c|c|c|c|c|c|}
\hline Order \# & $Q_{i}($ sets $)$ & $T P E_{i}$ & $T P E U_{i}$ & $L T_{i}$ & $Q_{i} L T_{i}$ & $\left(L T_{\mathrm{mw}}-L T_{i}\right)^{2}$ & $\left(L T_{\mathrm{mw}}-L T_{i}\right)^{2} Q$ \\
\hline 51 & 20 & $08 / 02$ & $25 / 02$ & 17 & 340 & 31.2 & 624.0 \\
\hline 52 & 20 & 08/02 & $26 / 02$ & 18 & 360 & 43.4 & 867.4 \\
\hline 53 & 20 & $13 / 02$ & $15 / 02$ & 2 & 40 & 88.6 & 1772.6 \\
\hline 54 & 40 & $13 / 02$ & $26 / 02$ & 13 & 520 & 2.5 & 100.6 \\
\hline 55 & 30 & $13 / 02$ & $27 / 02$ & 14 & 420 & 6.7 & 200.6 \\
\hline 56 & 40 & $13 / 02$ & $25 / 02$ & 12 & 480 & 0.3 & 13.7 \\
\hline 57 & 40 & $13 / 02$ & $21 / 02$ & 8 & 320 & 11.7 & 466.3 \\
\hline 58 & 60 & $18 / 02$ & $26 / 02$ & 8 & 480 & 11.7 & 699.5 \\
\hline 59 & 40 & $18 / 02$ & $26 / 02$ & 8 & 320 & 11.7 & 466.3 \\
\hline 60 & 20 & $18 / 02$ & $25 / 02$ & 7 & 140 & 19.5 & 389.7 \\
\hline 61 & 40 & $18 / 02$ & $26 / 02$ & 8 & 320 & 11.7 & 466.3 \\
\hline 62 & 20 & $18 / 02$ & $26 / 02$ & 8 & 160 & 11.7 & 233.2 \\
\hline 63 & 30 & $19 / 02$ & $26 / 02$ & 7 & 210 & 19.5 & 584.6 \\
\hline 64 & 20 & $20 / 02$ & $05 / 03$ & 13 & 260 & 2.5 & 50.3 \\
\hline 65 & 20 & $21 / 02$ & $27 / 02$ & 6 & 120 & 29.3 & 586.3 \\
\hline 66 & 20 & $21 / 02$ & $11 / 03$ & 18 & 360 & 43.4 & 867.4 \\
\hline 67 & 50 & $21 / 02$ & $11 / 03$ & 18 & 900 & 43.4 & 2168.5 \\
\hline 68 & 90 & $21 / 02$ & $12 / 03$ & 19 & 1710 & 57.5 & 5178.7 \\
\hline 69 & 50 & $22 / 02$ & $04 / 03$ & 10 & 500 & 2.0 & 100.0 \\
\hline 70 & 40 & $22 / 02$ & $05 / 03$ & 11 & 440 & 0.2 & 6.9 \\
\hline 71 & 50 & $25 / 02$ & $28 / 02$ & 3 & 150 & 70.8 & 3540.1 \\
\hline 72 & 20 & $27 / 02$ & $12 / 03$ & 13 & 260 & 2.5 & 50.3 \\
\hline 73 & 20 & $28 / 02$ & $06 / 03$ & 6 & 120 & 29.3 & 586.3 \\
\hline 74 & 20 & $28 / 02$ & $06 / 03$ & 6 & 120 & 29.3 & 586.3 \\
\hline 75 & 80 & $01 / 03$ & $19 / 03$ & 18 & 1440 & 43.4 & 3469.6 \\
\hline 76 & 20 & $01 / 03$ & $07 / 03$ & 6 & 120 & 29.3 & 586.3 \\
\hline 77 & 30 & $04 / 03$ & $06 / 03$ & 2 & 60 & 88.6 & 2658.9 \\
\hline 78 & 20 & $04 / 03$ & $22 / 03$ & 18 & 360 & 43.4 & 867.4 \\
\hline 79 & 60 & $05 / 03$ & $22 / 03$ & 17 & 1020 & 31.2 & 1871.9 \\
\hline 80 & 20 & $06 / 03$ & $12 / 03$ & 6 & 120 & 29.3 & 586.3 \\
\hline 81 & 50 & $06 / 03$ & $11 / 03$ & 5 & 250 & 41.1 & 2057.3 \\
\hline 82 & 50 & $06 / 03$ & $20 / 03$ & 14 & 700 & 6.7 & 334.3 \\
\hline 83 & 30 & $07 / 03$ & $13 / 03$ & 6 & 180 & 29.3 & 879.5 \\
\hline 84 & 40 & $07 / 03$ & $14 / 03$ & 7 & 280 & 19.5 & 779.5 \\
\hline 85 & 20 & $07 / 03$ & $11 / 03$ & 4 & 80 & 55.0 & 1099.5 \\
\hline 86 & 40 & $11 / 03$ & $13 / 03$ & 2 & 80 & 88.6 & 3545.3 \\
\hline 87 & 50 & $11 / 03$ & $13 / 03$ & 2 & 100 & 88.6 & 4431.6 \\
\hline 88 & 20 & $11 / 03$ & $21 / 03$ & 10 & 200 & 2.0 & 40.0 \\
\hline 89 & 20 & $11 / 03$ & $22 / 03$ & 11 & 220 & 0.2 & 3.4 \\
\hline 90 & 30 & $12 / 03$ & $26 / 03$ & 14 & 420 & 6.7 & 200.6 \\
\hline 91 & 30 & $12 / 03$ & $27 / 03$ & 15 & 450 & 12.9 & 385.7 \\
\hline 92 & 20 & $12 / 03$ & $27 / 03$ & 15 & 300 & 12.9 & 257.1 \\
\hline 93 & 30 & $13 / 03$ & $28 / 03$ & 15 & 450 & 12.9 & 385.7 \\
\hline 94 & 30 & $13 / 03$ & $27 / 03$ & 14 & 420 & 6.7 & 200.6 \\
\hline 95 & 50 & $15 / 03$ & $19 / 03$ & 4 & 200 & 55.0 & 2748.7 \\
\hline 96 & 20 & $15 / 03$ & $27 / 03$ & 12 & 240 & 0.3 & 6.9 \\
\hline 97 & 30 & $18 / 03$ & $26 / 03$ & 8 & 240 & 11.7 & 349.8 \\
\hline 98 & 30 & $18 / 03$ & $01 / 04$ & 14 & 420 & 6.7 & 200.6 \\
\hline 99 & 40 & $18 / 03$ & $27 / 03$ & 9 & 360 & 5.8 & 233.2 \\
\hline 100 & 30 & $20 / 03$ & $03 / 04$ & 14 & 420 & 6.7 & 200.6 \\
\hline
\end{tabular}

TABle 1. Raw data and the model application. Part 2/2. 


\begin{tabular}{ccc}
\hline $\begin{array}{c}\text { Time } \\
\text { interval }\end{array}$ & $\begin{array}{c}\text { Proportion } \\
\left(p_{n}\right)\end{array}$ & Accumulated \\
\hline 0 & $58 \%$ & $58 \%$ \\
1 & $25 \%$ & $83 \%$ \\
2 & $6 \%$ & $89 \%$ \\
3 & $7 \%$ & $96 \%$ \\
4 & $1 \%$ & $97 \%$ \\
5 & $3 \%$ & $100 \%$ \\
\hline
\end{tabular}

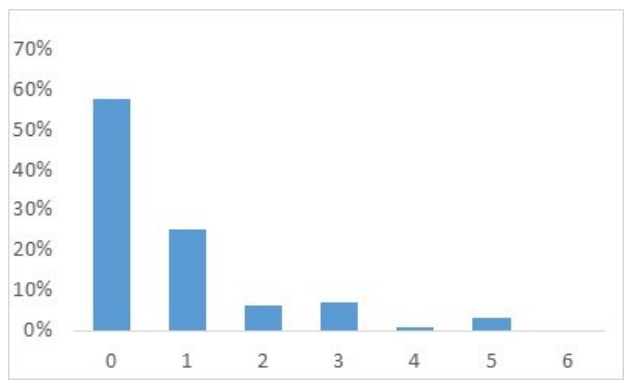

FIGURE 2. Distribution of the time intervals between arrivals of orders.

\begin{tabular}{cllr}
\hline Variable & Result & Unit & Equation \\
\hline$L T_{\mathrm{m}}$ & $=10.9$ & days & $\sqrt{2}$ \\
$L T_{\sigma \mathrm{m}}$ & $=5.8$ & days & \\
$L T_{\mathrm{mw}}$ & $=11.4$ & days & \\
$L T_{\sigma \mathrm{w}}$ & $=5.6$ & days & \\
\hline$R I_{\mathrm{m}}$ & $=48.6$ & sets/day & \\
$W I P_{\mathrm{m}}$ & $=554.4$ & sets & \\
$P_{\mathrm{m}}$ & $=41.6$ & sets $/$ day & \\
$S S_{\mathrm{m}}$ & $=207.8$ & sets &
\end{tabular}

TABLE 2. Results of the application.

\section{Discussion}

The mean order $L T$ and mean part $L T$ are close (10.9 and 11.4 days respectively), but not equal due to the variability in the order size $(\mu=37.4, \sigma=18.4$ sets $)$. To cope with this uncertainty, the model creates the bivariate stochastic variable $L T_{i} Q_{i}$, without a direct physical meaning that represents the use of the manufacturing system by an order. The rationale that explains $L T_{i} Q_{i}$ is: the smaller the time to completion or the order size, the smaller the use of the system; the larger the time to completion or the order size, the larger the use of the system. The sum $\sum_{i=1}^{n} L T_{i} Q_{i}$ is a proxy variable representing the total use of the system in the period. The division of $\sum_{i=1}^{n} L T_{i} Q_{i}$ by the total number of parts produces a proxy variable that represents the expected time to completion of a single part.

The mean arrival rate $R I_{\mathrm{m}}$ is 48.6 sets per day. The mean throughput $P_{\mathrm{m}}$ is 41.6 sets per day. Since $P_{\mathrm{m}}$ is lower than $R I_{\mathrm{m}}$, the manufacturing system accumulated an inventory in the period. The mean inventory $W I P_{\mathrm{m}}$ is 554.4 sets of parts, which is more than two times the required buffer number of sets $\left(S S_{\mathrm{m}}=207.3\right)$ and ensures an excessive protection against a starvation. A reduction on the buffer would result in a much lower inventory cost, as it is usually required by manufacturing strategies. In the period, the maximum interval between two arrivals is five days, in three out of the 100 orders. Assuming a normal distribution for the inventory, a $W I P_{\mathrm{m}}$ of 207.3 sets provides a $50 \%$ protection for the three orders with inter-arrival times of five days. For a full protection for the orders with inter-arrival times lower than five days, the upper limit for the safety level $S L$, the probability that the manufacturing system will not be stopped by starvation, is $S L=1-0.5 \cdot 3 / 100=$ $98.5 \%$, which is too high for the manufacturing purposes. The $S L$ is expected to decrease when taking into account the inventory uncertainty.

Figure 2 shows the data and the distribution of the inter-arrival times. The data follow a negative exponential distribution.

Another usual requirement of a manufacturing strategy is the full attendance of the orders due dates, which requires managing the lead-time. The maximum order lead-time is 28 days. Therefore, promised dates of 28 or more days ensure a full compliance with due dates. On the one hand, a promised date of 28 days satisfactorily protects the majority of orders; on the other hand, it could jeopardize a fast-delivery sales policy. A compromise solution, a trade-off between the protection and speed is needed. Considering $L T_{\mathrm{m}}=10.9$ and $L T_{\sigma \mathrm{m}}=5.8$ days, the upper limit of a $95 \%$ confidence interval for the order lead-time is $L T_{U L}=10.9+1.96 \cdot 5.8=22.3$ days. A promised date of 23 days would protect $95 \%$ of the sales. If the sales policy requires deadlines of no more than 20 days, the safety level would be $S L=1-N(20,10.9,5.8)=94.2 \%$, which usually suffices for a competitive manufacturing strategy.

The next stage is the use the TD to graphically verify the analytic calculation of $L T_{\mathrm{m}}$ and $I_{\mathrm{m}}$. Figure 3 shows the inflow and outflow TD and regression models.

The figure shows the accumulation of arrivals and completion of orders in the period, the regression models and the respective $R^{2}$. The figure shows two models for the outflow, one with the intercept that maximizes the fitting, the other with the intercept equal to zero. The reason is that the negative intercept that maximizes $R^{2}$ has no physical meaning and harms the analysis, distorting the angular coefficient. The first regression model serves to verify the calculation of $L T_{\mathrm{m}}$ and $W I P_{\mathrm{m}}$. The second serves to verify $P_{\mathrm{m}}$. As both $R^{2}$ are close to 1 , the models can satisfactorily replace the raw data.

Regarding $R I$ and $P$, the slopes are close to the analytical outcomes $\left(R I_{\mathrm{m}}=48.8\right.$ and $48.6 ; P_{\mathrm{m}}=41.6$ and 41.04), which reinforces the validity of the model. In the long term, the manufacturing control releases on average 48.8 parts per day to the production line that dispatches on average 41.6 parts per day, producing an increasing instantaneous $W I P$ and a $W I P_{\mathrm{m}}$ larger than the minimum needed to avoid a starvation, the $S S_{\mathrm{m}}$. 


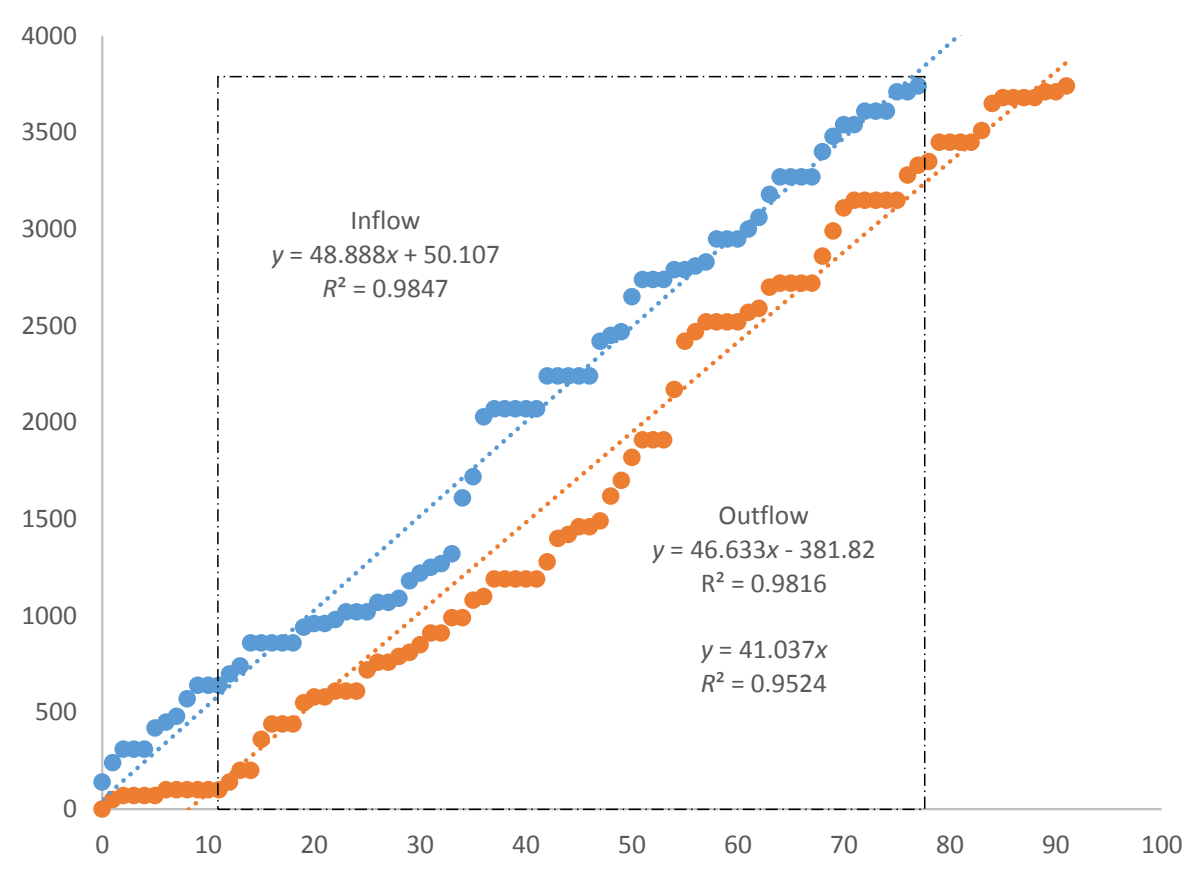

Figure 3. The TD and the regression models.

To reduce the excess, the manufacturing control should slow the order release, increase the processing capacity, or both, until $W I P_{\mathrm{m}}$ meets $S S_{\mathrm{m}}$.

Regarding $L T$ and $W I P$, the central points of the regression models are $(x, y)=(41.5,1870)$, corresponding respectively to the centre of time and inventory ranges. The horizontal distance between the inflow and outflow model at $y=1870$ and the vertical distance at $x=45.5$ represent $L T_{\mathrm{m}}$ and $W I P_{\mathrm{m}}$ respectively. After some algebraic manipulation with the equations of the two regression models, it is possible to calculate the horizontal and the vertical distance between the central points of the lines. $L T_{\mathrm{m}}=\frac{1870+381.8}{46.6}-\frac{1870-50.1}{48.8}=11.02$ days corresponds to the horizontal distance. $W I P_{\mathrm{m}}=$ $(48.8 \cdot 45.5+50.1)-(46.6 \cdot 45.5-381.8)=532$ sets corresponds to the vertical distance between the central points of the lines. Again, the graphical values are close (11.4 and $11.02 ; 554.4$ and 532) to the analytical outcomes of the model. As the variables are stochastic, the approximations suffice for the analysis.

Finally, the TD also allows considering the inventory uncertainty. The orders arrived in 77 days, but only those inside the dashed area in Figure 3 are useful. The analysis discards the initial orders, as they require negative outflow accumulation to calculate the instantaneous inventory, which has no physical meaning. The mean and the standard deviation of the 67 valid instantaneous inventory values are 548.4 and 202.1 sets respectively. Assuming a fixed value $P_{\mathrm{m}}=41.04, W I P_{\mathrm{m}}=548.4$ and $W I P_{\sigma}=202.1$, and using $p_{n}$, the proportion of orders whose inter-arrival time is $n=1, \ldots, 5$, $S L=1-\sum_{n=1}^{5} p_{n} N(41.04 n, 548,202)=99.3 \%$, which is very high and confirms the excess of the inventory. If the management reduces the $W I P_{\mathrm{m}}$ to 207 sets, the analytical $S S_{\mathrm{m}}$, the new value for the standard de- viation is $W I P_{\sigma}=202.1 \sqrt{207 / 542.8}=124.15$ and $S L=1-\sum_{n=1}^{5} p_{n} N(41.04 n, 207,124)=93.2 \%$, which is still acceptable in a competitive manufacturing strategy. As expected, the uncertainty reduced the $S L$, whose upper limit was previously estimated at $98.5 \%$.

\section{Conclusion}

The purpose of this article was to present a method for calculating lead-time, inventory, and the safety stock in job shop MTO manufacturing. The article included an application of the model in a job shop MTO manufacturing of the furniture industry. Besides the calculation of the variables, the application demonstrated that, due to unbalanced flows of material (arrival and completion of orders), the manufacturing produced excessive $W I P$, which possibly impairs the delivery deadlines and increases the operational cost. As the manufacturing strategy requires reliability in deliveries, the management should focus on reducing $W_{\mathrm{m}}$ to ensure a dependability and support the competition in the industry.

The article presented the initial development of the study. Further research shall focus on the influence of the uncertainty of the order size and the inventory in the leadtime. The research method is the simulation, which is widely used in manufacture control studies 14. Further research shall also include fuzzy sets theory to manage the intrinsic uncertainty in the inventory management. A systematic review identified major achievements in fuzzy inventory management [15]. Further research should also focus on other companies in the same industry and in other industries, such as the footwear and the electronics industries that also rely on MTO job shop manufacturing systems. 


\section{REFERENCES}

[1] Thürer M, Stevenson M, Land M. On the integration of input and output control: Workload Control order release. Int J Prod Econ 2016; 174:43-53. DOI:10.1016/j.ijpe.2016.01.005

[2] Fernandes N., Land M., Carmo-Silva S. Workload control in unbalanced job shops. Int J Prod Rese 2014; 52: 679-690. DOI:10.1080/00207543.2013.827808

[3] Sellitto M, Luchese J. Systemic cooperative actions among competitors: the case of a furniture cluster in Brazil. Journal of Industry, Competition and Trade (accepted, on line first) DOI:10.1007/s10842-018-0272-9

[4] Sellitto M, Luchese J, Bauer J, Saueressig G, Viegas C (2017). Ecodesign Practices in a Furniture Industrial Cluster of Southern Brazil: From Incipient Practices to Improvement. Journal of Environmental Assessment Policy and Management 2017; 19:

1750001 DOI:10.1142/S1464333217500016

[5] Henrich P, Land M, Gaalman G. Grouping machines for effective workload control. Int J Prod Econ 2006; 104:125-142. DOI:10.1016/j.ijpe.2004.11.006

[6] Stevenson M, Huang Y, Hendry L, Soepenberg E. The theory and practice of workload control: A research agenda and implementation strategy. Int J Prod Econ 2011; 131:689-700. DOI:10.1016/j.ijpe.2011.02.018

[7] Hendry L, Land M, Stevenson, M., Gaalman, G. (2008). Investigating implementation issues for workload control (WLC): a comparative case study analysis. Int J Prod Econ 2008; 112:452-469. DOI:10.1016/j.ijpe.2007.05.012

[8] Perona M, Saccani N, Bonetti S, Bacchetti A. Manufacturing lead time shortening and stabilisation by means of workload control: an action research and a new method. Prod Plan Control 2016; 27: 660-670. DOI:10.1080/09537287.2016.1166283
[9] Wiendahl H, Glässner J, Petermann D. Application of load-oriented manufacturing control in industry. Prod Plan Control 1992; 3:118-129 DOI:10.1080/09537289208919381

[10] Haskose A, Kingsman B, Worthington, D. Performance analysis of make-to-order manufacturing systems under different workload control regimes. Int J Prod Econ 2004; 90:169-186 DOI:10.1016/S0925-5273(03)00052-5

[11] Bechte, W. Theory and practice of load-oriented manufacturing control. Int J Prod Rese 1988; 26:375-395 DOI:10.1080/00207548808947871

[12] Land M, Stevenson M, Thürer M. Integrating load-based order release and priority dispatching. Int J Prod Rese 2014; 52:1059-1073. DOI:10.1080/00207543.2013.836614

[13] Thürer M, Silva C, Stevenson M, Land, M. Improving the applicability of workload control (WLC): the influence of sequence-dependent set-up times on workload controlled job shops. Int J Prod Rese 2012; 50: 6419-6430. DOI:10.1080/00207543.2011.648275

[14] Thürer M., Stevenson, M. Workload control in job shops with re-entrant flows: an assessment by simulation. Int J Prod Rese 2016; 54: 5136-5150. DOI:10.1080/00207543.2016.1156182

[15] Shekarian E., Kazemi N., Abdul-Rashid, S., Olugu, E. Fuzzy inventory models: A comprehensive review. Appl Soft Comput 2017; 55: 588-621. DOI:10.1016/j.asoc.2017.01.013

[16] Michalski, G. Value Maximizing Corporate Current Assets and Cash Management in Relation to Risk Sensitivity: Polish Firms Case. Econ Comput Econ Cyb 2014; 48:259-276. DOI:10.2139/ssrn.2442862

[17] Michalski, G. Full Operating Cycle Influence on Food and Beverages Processing Firms Characteristics. Agric Econ 2016; 62:71-77 DOI:10.17221/72/2015-AGRICECON

[18] Michalski, G. (2016). Risk pressure and inventories levels. Influence of risk sensitivity on working capital levels. Econ Comput Econ Cyb 2016; 50:189-196. 